ISSN 1112-9867

http://www.jfas.info

\title{
MHD FREE CONVECTION FLOW OF A NON-NEWTONIAN POWER-LAW FLUID OVER A VERTICAL PLATE WITH SUCTION EFFECTS
}

\author{
E. Mahmoudpour Molaei ${ }^{1, *}$ and F. Rouzbahani ${ }^{2}$ \\ ${ }^{1}$ Department of Mechanical Engineering, Brojerd Branch, Islamic Azad University, Brojerd, \\ Iran \\ ${ }^{2}$ Department of Mechanical Engineering, Hamedan Branch, Islamic Azad University, \\ Hamedan, Iran
}

Published online: 09 June 2016

\begin{abstract}
In this study, we present a numerical analysis of free convection flow and heat transfer is presented for non-Newtonian power-law fluids with MHD effects over a vertical porous plate, the surface of which is exposed to a constant wall temperature. For analysis, the Continuty, Momentum and Energy equations are solved by the implicit finite difference scheme of Crank-Nicolson's type. Velocity and temperature of the flow have been presented for various parameters such as Prandtl number, flow behavior index (n), porous plate parameter and magnetic parameter. The local Nusselt number and skin friction coefficient is also presented graphically.
\end{abstract}

Keywords: Magnetohydrodynamic flow; free convection flow; Non-Newtonian power-law fluids; Vertical porous plate.

Author Correspondence, e-mail: E.mahmoodpour@yahoo.com

doi: http://dx.doi.org/10.4314/jfas.8vi2s.65 


\section{INTRODUCTION}

The In recent years MHD flow and non-Newtonian fluid behavior problems have become in view of its significant applications in industrial manufacturing processes such as plasma studies, petroleum industries Magneto-hydrodynamics power generator cooling of clear reactors, boundary layer control in aerodynamics. Many authors have studied the effects of magnetic field on mixed, natural and force convection heat and mass transfer of non-Newtonian fluid problems. Magnetohydrodynamic has attracted the attention of a large number of scholars due to its diverse applications. In astrophysics and geophysics, it is applied to study the stellar and solar structures, interstellar matter, radio propagation through the ionosphere etc. In engineering it finds its application in MHD pumps, MHD bearings etc. Convectionon non-Newtonian fluid in porous media has applications in geothermal energy recovery, oil extraction, thermal energy storage and flow through filtering devices .The phenomena of mass transfer is also very common in theory of stellar structure and observable effects are detectable, at least on the solar surface. The study of effects of magnetic field on free convection flow of non-Newtonian fluid is important in liquid-metals, electrolytes and ionized gases. The thermal physics of hydromagnetic problems with mass transfer is of interest in power engineering and metallurgy.

Pop and $\mathrm{Na}$ [1] presented a case of free convection heat transfer of non-Newtonian fluids along a vertical wavy surface in a porous medium. Yue-Tzu and Wang [2] studied the problem of natural convection of a non-Newtonian power-law fluid with and without yield stress about a two- dimensional axi-symmetric body of arbitrary shape in a fluid- saturated porous medium on the basis of boundary layer approximation. Chen and Chen [3] presented similarity solutions for natural convection of a non-Newtonian fluid over vertical surfaces in porous media. Jumah and Mujumder [4] investigated the problem of natural convection coupled heat and mass transfer from a vertical flat plate in a porous medium saturated with a Herschel Bulkley fluids. Abel and Veena [5] studied the viscoelasticity on the flow and heat transfer in a porous medium over a stretching sheet. Bestman [6] examined the natural convection boundary layer with suction and mass transfer in a porous medium. Comparatively little work exists on non- Newtonian fluid-saturated porous media as is evident from the review by 
Shenoy [7] .Makinde [8] examined the transient free convection interaction with thermal radiation of an absorbing-emitting fluid along moving vertical Permeable plate. Chamkha [9] assumed that the plate is embedded in a uniform porous medium and moves with a constant velocity in the flow direction in the presence of a transverse magneticeld. Kim [10] studied unsteady MHD convective heat transfer past a semi-infinite vertical porous moving plate. ELHakiem et al .[11] presented group theoretical analysis of unsteady free convection flow over a continuous moving vertical plate embedded in a fluid-saturated porous medium in the presence of amagnetic field .M.A.Abd El-Naby et al. [12] presented Finite difference solution of radiation Effects on MHD unsteady free-convection flow over vertical porous plate.

The object of the present paper is to study the transient free convection and heat transfer flow of non-Newtonian power-law fluids past a vertical porous plate, under the influence of a uniform transverse magnetic field in the presence of constant wall temperature. The dimensionless governing equations are solved by using implicit finite difference scheme of Crank-Nicolson's type. Velocity and temperature of the flow have been presented for various parameters such as Prandtl number, flow behavior index (n), porous plate parameter and magnetic parameter. The local Nusselt number and skin friction coefficient is also presented graphically.

\section{Nomenclature}

$\mathrm{B}_{0} \quad$ Magnetic field flux density

g Acceleration due to gravity

$t^{\prime} \quad$ Dimensionless time

K Thermal conductivity

t Time

n The flow behavior index

T Temperature of the fluid

$\mathrm{u}, \mathrm{v} \quad$ Velocity components along(x,y)-axis

U,V Dimensionless velocity components in

X,Y Directions respectively

\section{Greek symbols}

$\tau \quad$ Shear stress

$\bar{\mu} \quad$ Dimensionless of dynamic viscosity

$\beta \quad$ Coefficient of expansion

$\mu \quad$ Coefficient of dynamic viscosity

$\mu_{o} \quad$ Reference dynamic viscosity

$\sigma \quad$ Electrical conductivity of the fluid,

$\alpha \quad$ Thermal diffusivity

$\rho \quad$ Fluid density

v Kinematic viscosity

$v_{o} \quad$ Reference Kinematic viscosity 
L The length of the plate

x Special coordinate along the plate

X Dimensionless spatial coordinate along the plate

y Spatial coordinate normal to the plate

Y Dimensionless spatial coordinate normal to the plate W

Gr Grashof number

Pr Prandtl number

$\mathrm{Nu} \quad$ Nusselt number

W Evaluated at wall conditions

M Magnetic parameter $\theta \quad$ Dimensionless temperature

$\theta_{W} \quad$ Surface temperature ratio

\section{Subscripts}

Evaluated at wall conditions

$\infty \quad$ Evaluated at free stream condition

\section{MATHEMATICAL FORMULATION}

The Physical model, coordinate system and boundary conditions are shown in Fig .1. Consider a MHD free convection flow of an electrically conducting, for non-Newtonian power-law fluids over an isothermal vertical porous plate. The $\mathrm{x}$-axis is assumed to be taken along the plate and the $y$-axis normal to the plate. The wall is maintained at conestant temperature $T_{w}$ and than the ambient temperature $\mathrm{T}_{\infty}$ respectively .a uniform magnetic field is applied normal of magnitude $\mathrm{B}_{0}$ to the plate.

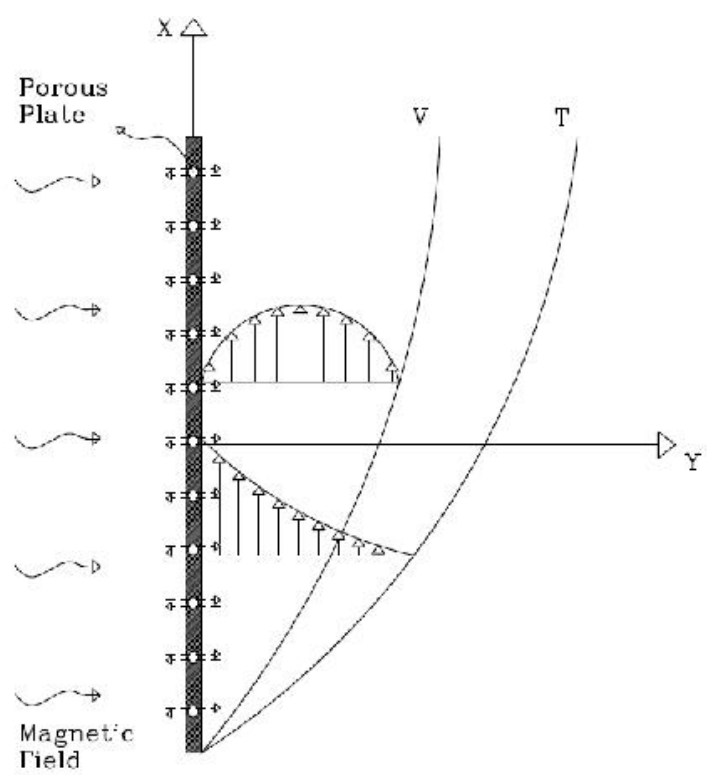

Fig.1. Physical model and coordinate system 
The governing equations include mass conservation in a continuous medium (Equ. 1), the Navier-Stokes equation (Equ. 2) and also the energy equation (Equ. 3).

$\frac{\partial u}{\partial x}+\frac{\partial v}{\partial y}=0$

$\frac{\partial u}{\partial t}+u \frac{\partial u}{\partial x}+v \frac{\partial u}{\partial y}=\frac{1}{\rho} \frac{\partial}{\partial y}\left[\mu \frac{\partial u}{\partial y}\right]+g \beta\left(T-T_{\infty}\right)-\frac{\sigma B_{0}^{2}}{\rho} u$

$\frac{\partial T}{\partial t}+u \frac{\partial T}{\partial x}+v \frac{\partial T}{\partial y}=\alpha \frac{\partial^{2} T}{\partial y^{2}}$

The non-Newtonian fluid model used in this study is the power-law model (Equ. 4).

$\tau=m\left|\frac{\partial u}{\partial y}\right|^{n}$

The Coefficient of dynamic viscosity, $\mu$ for power-law fluids is given by (Equ. 5).

$\mu=m\left|\frac{\partial u}{\partial y}\right|^{n-1}$

The physical problem assumes the following initial and boundary conditions (Equ. 6).

$$
\begin{array}{lll}
t \leq 0: u=0, v=0, T=T_{\infty} & \\
t>0: v=-v_{0} \quad, \quad u=0, T=T_{w} & \text { at } \quad \text { at } \quad x=0 \\
t>0: u=0, T=T_{\infty} & \text { at } y \rightarrow \infty
\end{array}
$$

Introducing the following dimensionless quantities (Equ. 7)

$$
\begin{aligned}
& x=\frac{X}{L}, \quad y=\frac{Y}{L} G r^{1 / 4}, \quad u=\frac{U L}{v_{o}} G r^{-1 / 2}, \quad v=\frac{V L}{v_{o}} G r^{-1 / 4}, \bar{\mu}=\frac{\mu}{\mu_{o}}, \\
& \bar{\mu}=\left|\frac{\partial U}{\partial Y}\right|^{n-1}, \mu_{o}=m\left(\frac{v_{o} G r^{\frac{3}{4}}}{L^{2}}\right)^{n-1}, t=\frac{v_{o} t^{\prime}}{L^{2}} G r^{1 / 2}, \quad \theta=\frac{T-T_{\infty}}{T_{w}-T_{\infty}}, \\
& G r=\frac{g \beta L^{3}\left(T_{w}-T_{\infty}\right)}{v_{o}^{2}}, \quad M=\frac{\sigma B{ }_{0}^{2} L^{2}}{\mu_{o} G r^{1 / 2}}, \operatorname{Pr}=\frac{v_{o}}{\alpha}, \mu=m\left|\frac{\partial u}{\partial y}\right|^{n-1}
\end{aligned}
$$

The governing equations can be rewritten in dimensionless form as follows:

$$
\frac{\partial U}{\partial X}+\frac{\partial V}{\partial Y}=0
$$




$$
\begin{aligned}
& \frac{\partial U}{\partial t^{\prime}}+U \frac{\partial U}{\partial X}+V \frac{\partial U}{\partial Y}=n \frac{\partial^{2} U}{\partial Y^{2}}\left|\frac{\partial U}{\partial Y}\right|^{n-1}+\theta-M \cdot U \\
& \frac{\partial \theta}{\partial t^{\prime}}+U \frac{\partial \theta}{\partial X}+V \frac{\partial \theta}{\partial Y}=\frac{1}{\operatorname{Pr}} \frac{\partial^{2} \theta}{\partial Y^{2}}
\end{aligned}
$$

The corresponding initial and boundary conditions in non-dimensional form are given by (Equ. 11).

$t^{\prime} \leq 0, \theta=0, V=0, U=0$

$t^{\prime}>0, U=0, V=-V_{0}, \theta=1 \quad$ at $\quad Y=0$

$$
\begin{array}{llr}
t^{\prime}>0, U=0, \theta=0 & \text { at } & X=0 \\
t^{\prime}>0, U \rightarrow 0, \theta=0 & \text { at } \quad Y \rightarrow \infty
\end{array}
$$

The local as well as Nnsselt number and skin friction coefficient in terms of dimensionless quantities are given by:

$$
\begin{aligned}
& N u=-\left.X \frac{\partial \theta}{\partial Y}\right|_{Y=0} \\
& C_{f}=\left|\frac{\partial U}{\partial Y}\right|^{n-1}\left(\frac{\partial U}{\partial Y}\right)_{Y=0}
\end{aligned}
$$

\section{NUMERICAL SOLUTION OF THE PROBLEM}

The governing equations (8-10) are unsteady, coupled and non-linear with boundary conditions. An implicit finite-difference technique of Crank-Nicolson has been employed to solve the nonlinear coupled equations, as described (Thomas algorithm) in Carnahan et al [13]. The finite difference equations corresponding to equations (8-10) are as follows:

$$
\begin{aligned}
& \frac{U_{i, j}^{n+1}-U_{i-1, j}^{n+1}+U_{i, j}^{n}-U_{i-1, j}^{n}}{2 \Delta X}+\frac{V_{i, j+1}^{n+1}-V_{i, j-1}^{n+1}+V_{i, j+1}^{n}-V_{i, j-1}^{n}}{4 \Delta Y}=0 \\
& \frac{U_{i, j}^{n+1}-U_{i, j}^{n}}{\Delta t^{\prime}}+U_{i, j}^{n} \frac{U_{i, j}^{n+1}-U_{i-1, j}^{n+1}+U_{i, j}^{n}-U_{i-1, j}^{n}}{2 \Delta X}+v_{i, j}^{n} \frac{U_{i, j+1}^{n+1}-U_{i, j-1}^{n+1}+U_{i, j+1}^{n}-U_{i, j-1}^{n}}{4 \Delta Y} \\
& =n\left(\frac{U_{i, j+1}^{n+1}-2 U_{i, j}^{n+1}+U_{i, j-1}^{n+1}+U_{i, j+1}^{n}-2 U_{i, j}^{n}+U_{i, j-1}^{n}}{2 \Delta Y^{2}}\right)\left|\frac{U_{i, j+1}^{n+1}-U_{i, j-1}^{n+1}+U_{i, j+1}^{n}-U_{i, j-1}^{n}}{4 \Delta Y}\right|^{n-1} \\
& +\frac{\theta_{i, j}^{n+1}+\theta_{i, j}^{n}}{2}-M \frac{U_{i, j}^{n+1}+U_{i, j}^{n}}{2}
\end{aligned}
$$


$\frac{\theta_{i, j}^{n+1}-\theta_{i, j}^{n}}{\Delta t^{\prime}}+U_{i, j}^{n} \frac{\theta_{i, j}^{n+1}-\theta_{i-1, j}^{n+1}+\theta_{i, j}^{n}-\theta_{i-1, j}^{n}}{2 \Delta X}+v_{i, j}^{n} \frac{\theta_{i, j+1}^{n+1}-\theta_{i, j-1}^{n+1}+\theta_{i, j+1}^{n}-\theta_{i, j-1}^{n}}{4 \Delta Y}$

$=\frac{1}{\operatorname{Pr}} \frac{\theta_{i, j+1}^{n+1}-2 \theta_{i, j}^{n+1}+\theta_{i, j-1}^{n+1}+\theta_{i, j+1}^{n}-2 \theta_{i, j}^{n}+\theta_{i, j-1}^{n}}{2 \Delta Y^{2}}$

The region of integration is considered as a rectangle with sides $X_{\text {max }}(=1)$ and $Y_{\text {max }}(=10)$, where corresponding to $Y \rightarrow \infty$ which lies far from the momentum, energy and concentration boundary layers. An appropriate mesh sizes considered for the calculation are $\Delta X=0.01$, $\Delta Y=0.05$ and $\Delta t^{\prime}=0.005$.

\section{RESULTS AND DISCUSSIONS}

The transformed governing Eqs.(8), (9), (10) and the associated initial and boundary conditions (11) can be solved by the implicit finite difference scheme of Crank-Nicolson's type. In the paper we focused on the effects of the magnetic field parameter, porous plate parameter, Prandtl number and flow behavior index on the transient velocity and temperature profiles.

Figures .2 and 3, show the effect of the magnetic field parameter $\mathrm{M}$ on the velocity and temperature profile. It is observed that the velocity decreases with increases in M parameters. However, the Temperature increases with an increase in M parameters.

Figures .4 and 5, describe the behavior of transient velocity and temperature for fluids with different value of power-law index $n$. It can be observed that the temperature decreases monotonously with increasing the $n$ but the velocity has a different behavior for small and large $n$. 


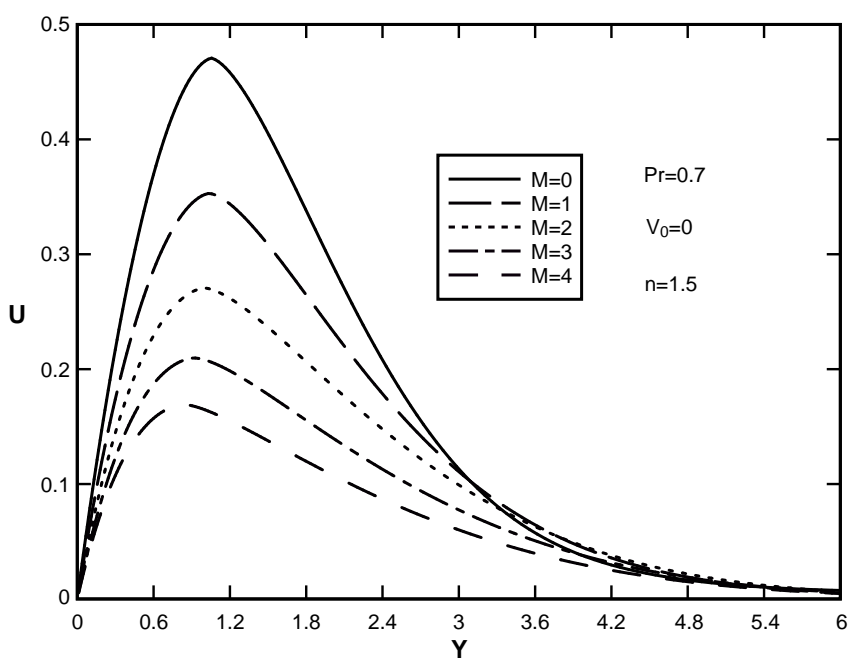

Fig.2. Transient velocity profiles for the different values of magnetic field parameter (M)

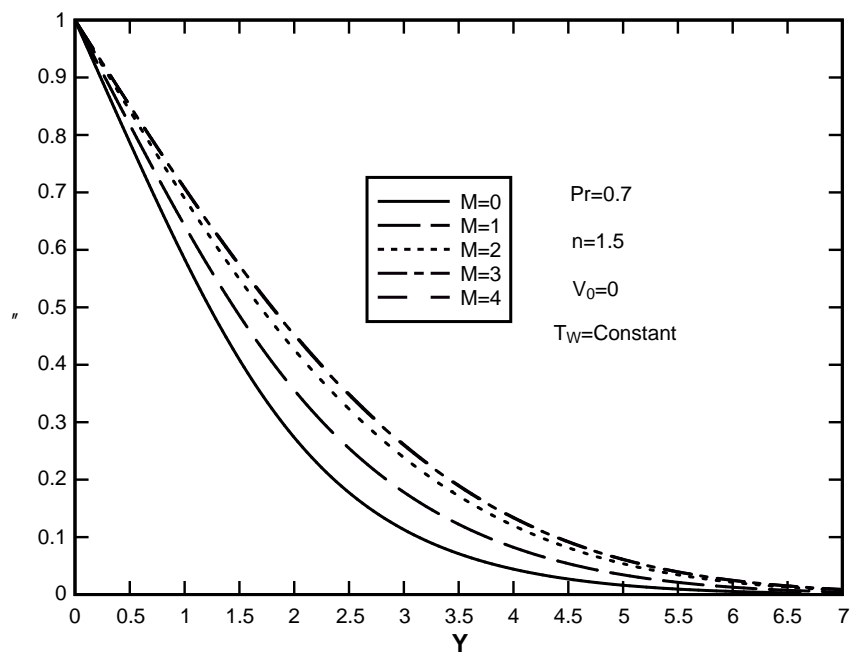

Fig.3. Transient temperature profiles for the different values of magnetic field parameter (M)

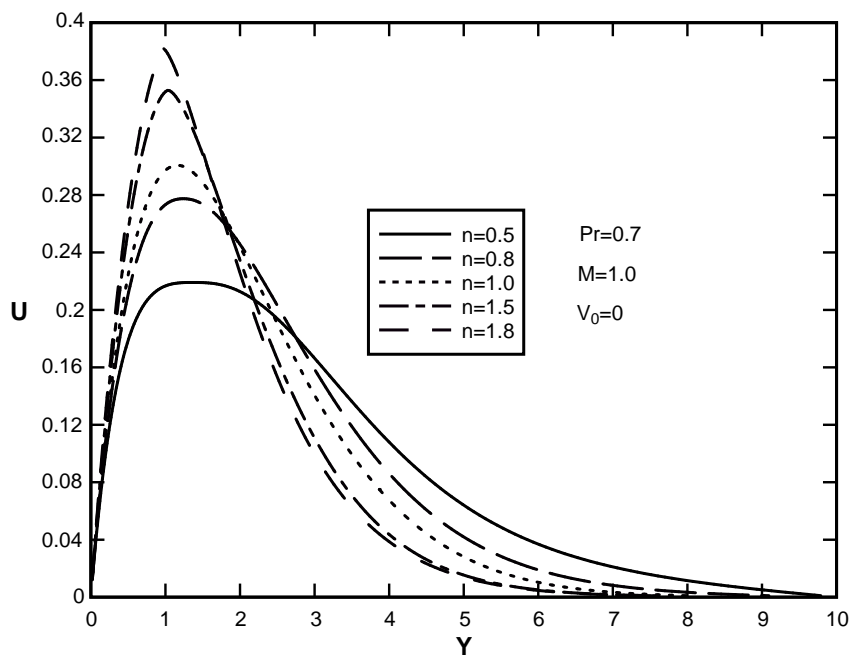

Fig.4. Transient velocity profiles for the different values of flow behavior index (n) 


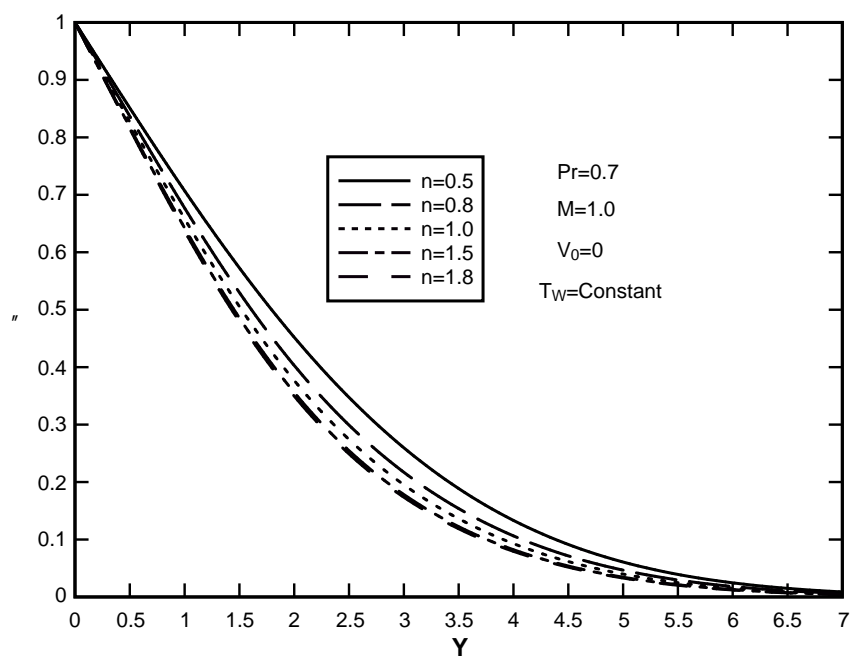

Fig.5. Transient temperature profiles for the different values of flow behavior index (n)

Figures.6 and 7, show the effect of Prandtl number on transient velocity and temperature distribution. Both velocity and temperature decrease as Prandtl number increases. This is in a greement with the physical fact that the thermal boundary layer thickness decreases with increasing Prandtl number. Figures .8 and 9, show the effect of the porous plate parameter on the velocity and temperature profile. It is observed that the velocity and Temperature increases with increases in porous plate parameter.

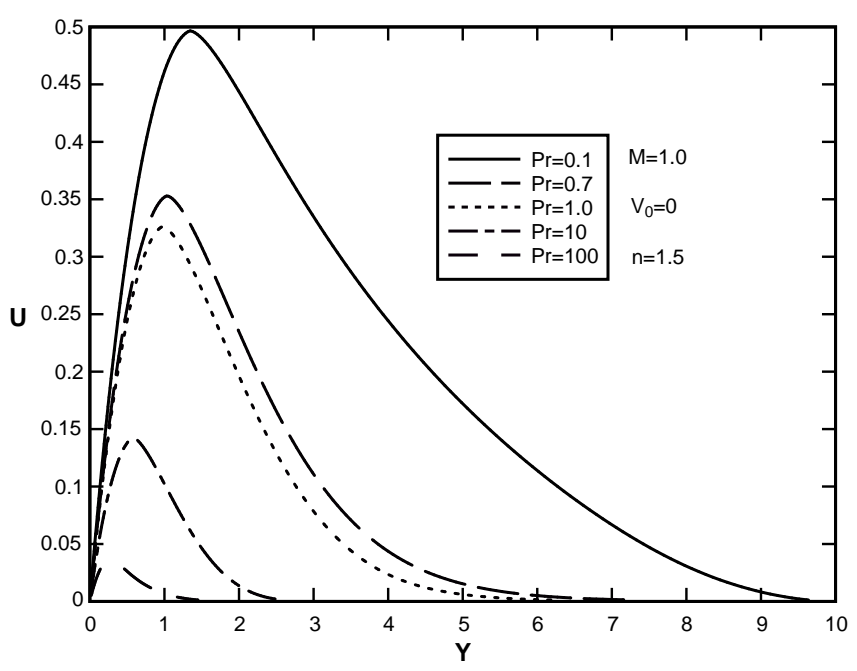

Fig.6. Transient velocity profiles for the different values of Prandtl number 


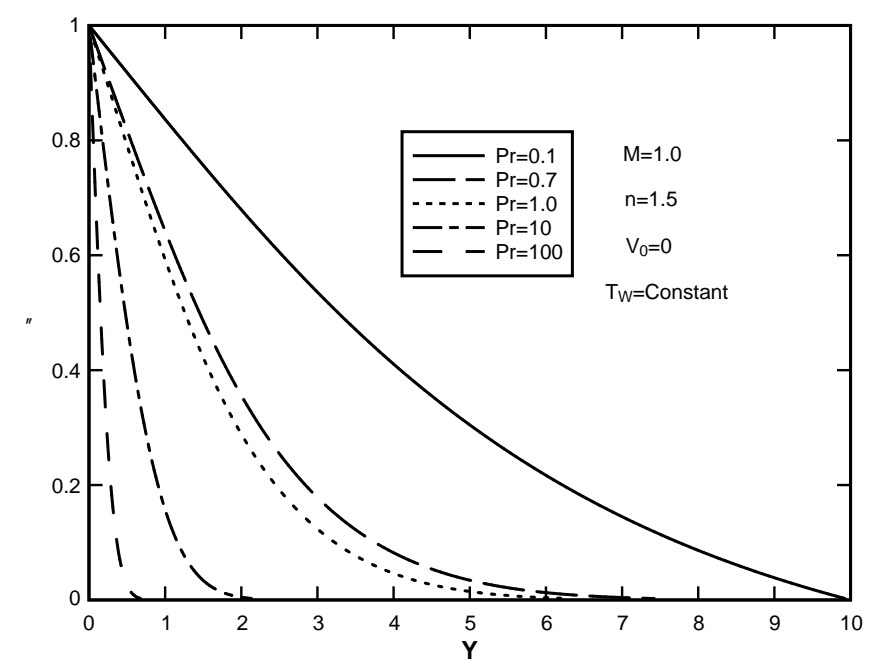

Fig.7. Transient temperature profiles for the different values of Prandtl number

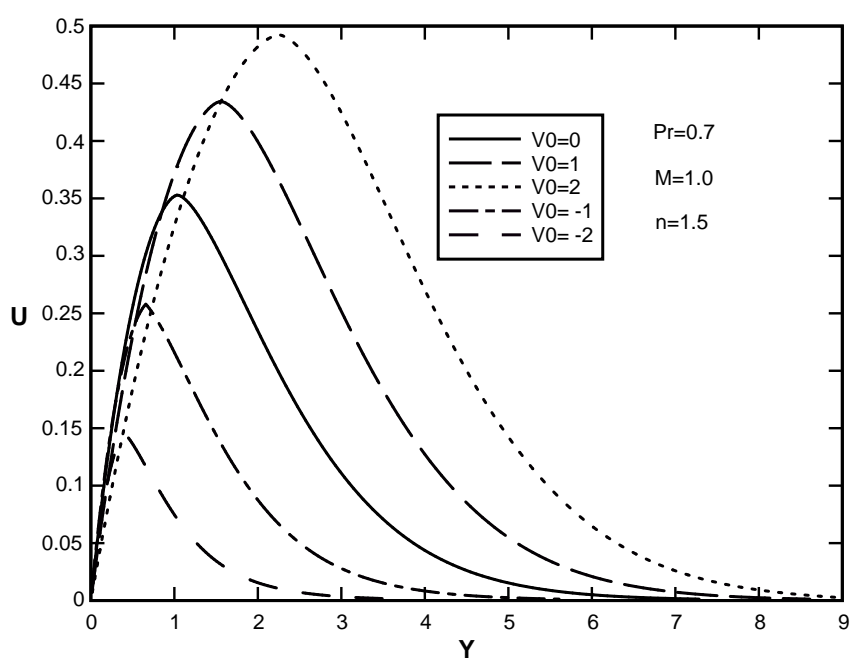

Fig.8. Transient velocity profiles for the different values of porous plate parameter

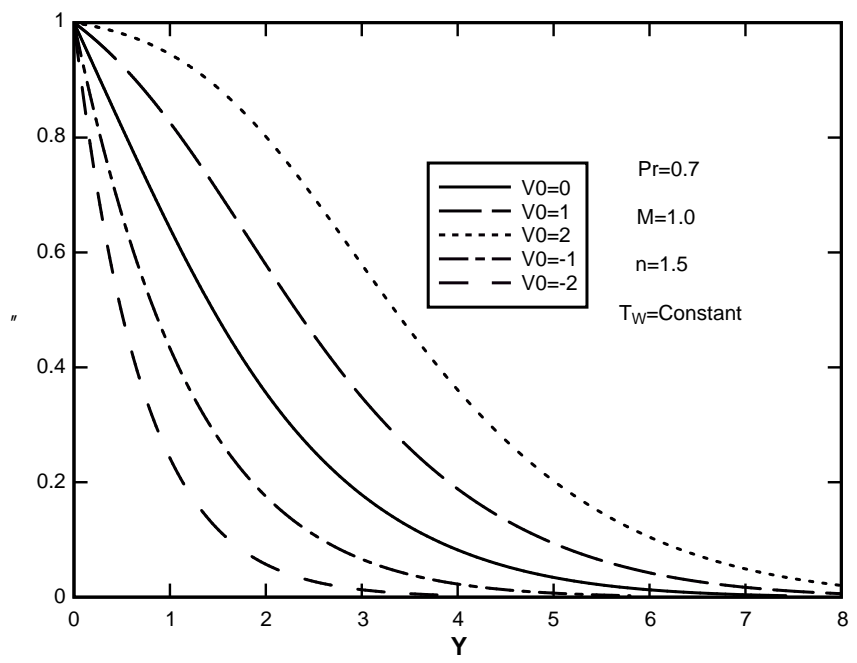

Fig.9. Transient temperature profiles for the different values of porous plate parameter 
Figures.10, show the effect of magnetic field parameter (M) on local Nusselt number respectively. It is observed that, local Nusselt number decrease as M increases.

Figure.11, show the effect of flow behavior index (n) on local Nusselt number respectively. It is observed that the local Nusselt number decrease with increases in flow behavior index.

Figures.12, show the effect of magnetic field parameter (M) on skin friction coefficient respectively. It is observed that, skin friction coefficient decrease as $\mathrm{M}$ increases.

Figure.13, show the effect of flow behavior index (n) on local skin friction coefficient respectively. It is observed that the skin friction coefficient increase with increases in flow behavior index.

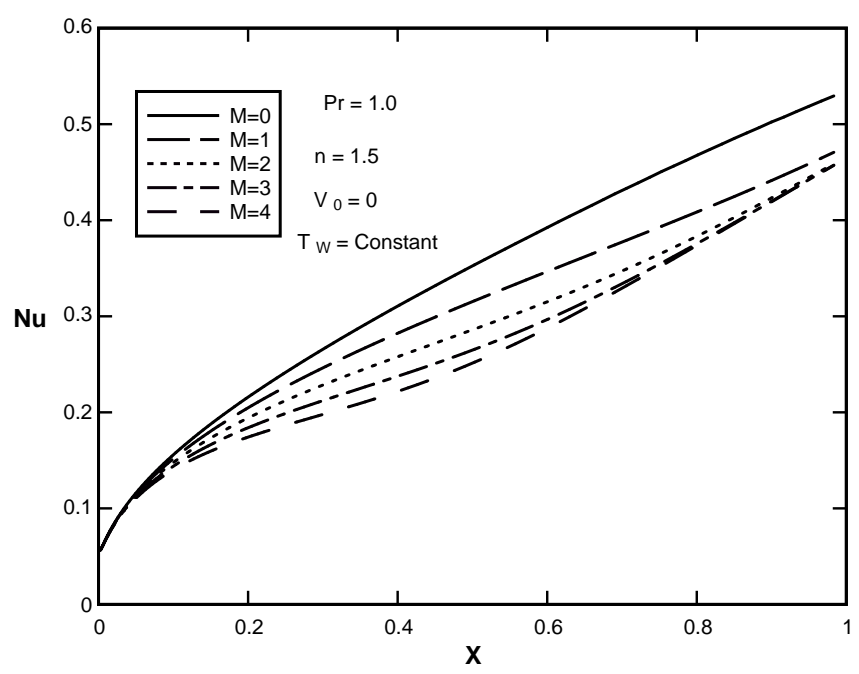

Fig.10. The effect of M parameter on the local Nusselt number

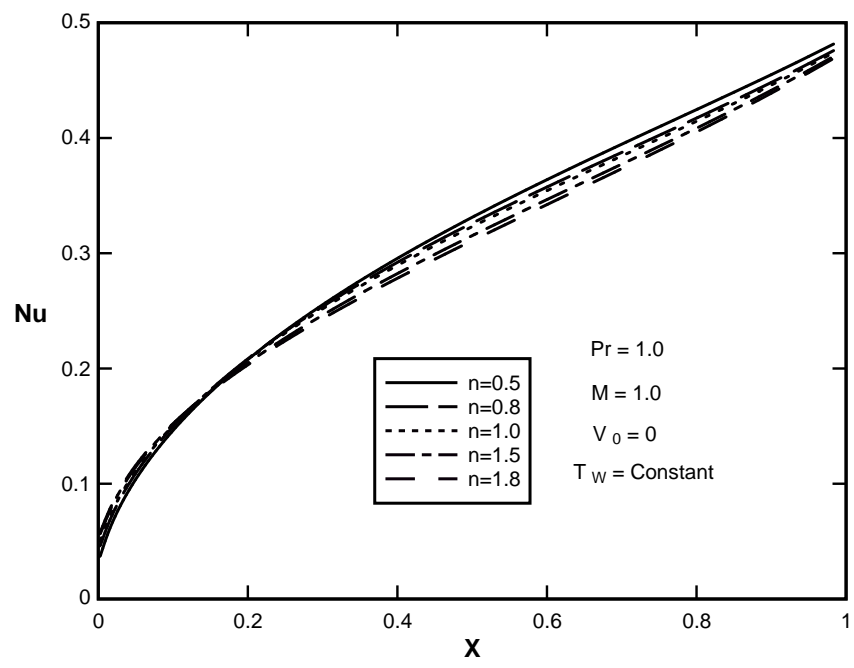

Fig.11. The effect of flow behavior index on the local Nusselt number 


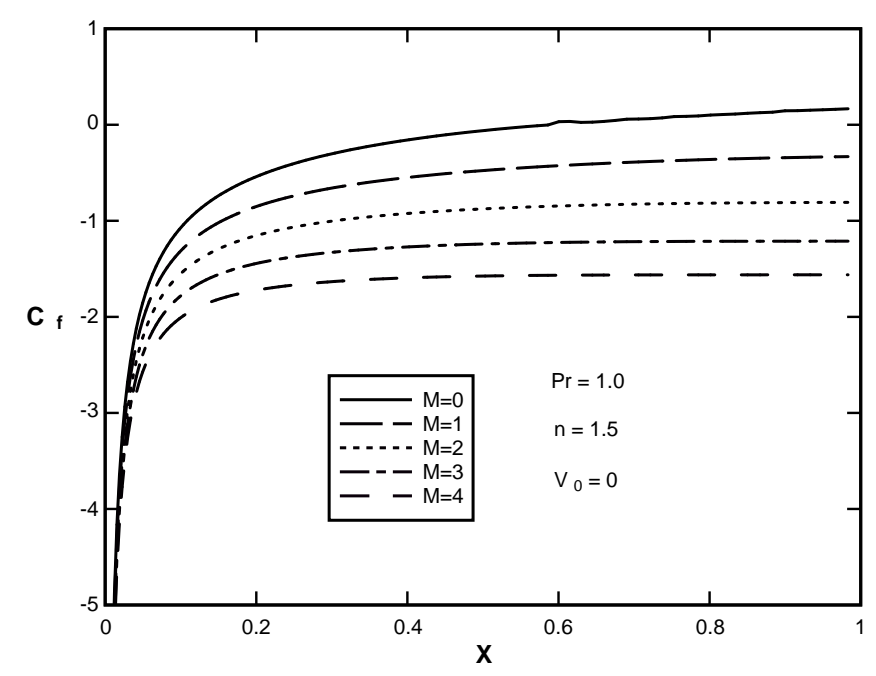

Fig.12. The effect of M parameter on the skin friction coefficient

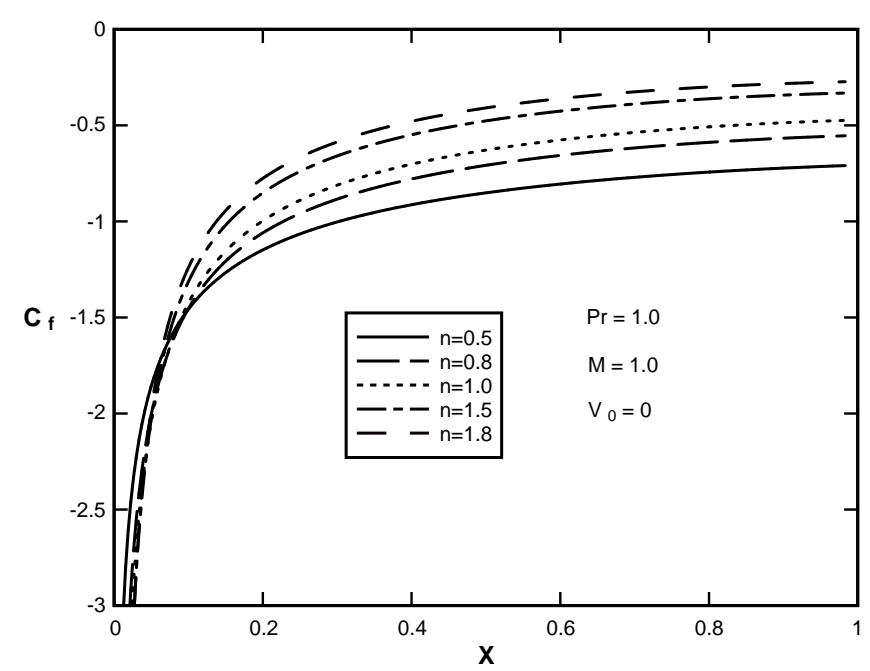

Fig.13. The effect of flow behavior index on the skin friction coefficient

\section{REFERENCES}

[1] Pop T and Na T Y. Free convection heat transfer of non-Newtonian fluids along a vertical wavy surface in a porous medium. Proceedings of the.4th International Symposium on Heat Transfer, Beijing, China, 1996, P452.

[2] Yue-Tzu Y and Wang S. Free convection heat transfer of non-Newtonian over axi-symmetric and two-dimensional bodies of arbitrary shape embedded in a fluidsaturated porous medium.International Journal of Heat and Mass Transfer, vol 39, no 1, 1996, p 203.

[3] Chen H. T, Chen C. K. Free convection of non-Newtonian fluids along a vertical plate 
embedded in a porous medium, ASME Journal of Heat Transfer 110, 1988, 257-260.

[4] Jumah R. Y, Mujumdar A. S. free convection heat and mass transfer of non-Newtonian power-law fluids with yield stress from a vertical flat plate in a saturated porous media , Int.Commun . Heat mass transfer, 2000, 27, 485-494.

[5] AbelS, Veena PH.Visco-elastic fluid flow and heat transfer in porous medium over astretching sheet. Int JNon-Linear Mech1 998;33:53140 [1] D.A. Nield and A. Bejan, Convection in Pourous Media, 2 nd edition p. 537, Springer, New York, 1999.

[6] Bestman A. R. Natural convection boundary layer with suction and mass transfer in a porous medium.Int J Energy Res 1990,14, 389-96.

[7] Shenoy A.V. Non-Newtonian Fluid Heat Transfer in Porous Media, In: J.P. Hartnett and T.F. IrvineJr (eds.), Advances in Heat Transfer, vol. 24, Academic Press, San Diego, 1994.

[8] Makinde O. D. Free convection flow with thermal radiation and mass transfer past a moving vertical porous plate. Int Comm Heat Mass Transfer, 2005, 32, 1411-9.

[9] Chamkha A. J .Unsteady MHD convective heat and mass transfer past a semi-innite vertical permeable moving plate with heat absorption.Int J Eng Sci, 2004, 42, 217-30.

[10] Kim Y. J. Unsteady MHD convective heat transfer rpast a semi-infinite vertical porous moving plate with variable suction, Internat .J. Engrg Sci., 2000, 38, 833-845.

[11]M.A.EL-Hakiem, S. M.M.EL-Kabeir, A.M.Rashad, Group method analysis of unsteady MHD natural convection flow over a moving vertical sheet in afluid saturated porous medium, J.Comput. Appl .Math. 2007, 384-397.

[12]Abd El-Naby M. A, Elsayed M.E. Elbarbary, AbdElazem Nader Y. Finite difference solution of radiation Effects on MHD unsteady free-convection flow over vertical porous plate. Int Comm Mathematics and computation, 2004, 151, 327-346.

[13]Carnahan B., Luther H. A. and Wilkes J. O. Applied Numerical Methods, Wiley, New York, 1969. 


\section{How to cite this article:}

Mahmoudpour Molaei E and Rouzbahani F. MHD Free convection flow of a non-newtonian power-law fluid over a vertical plate with suction effects

. J. Fundam. Appl. Sci., 2016, 8(2S), 487-500. 